\title{
MANSONI'S SCHISTOSOMIASIS OF A LEIOMYOMA: CASE REPORT
}

Raquel Martins Arruda, Rodrigo de Aquino Castro, Mariano Vieira Tamura, Jose María Ruano, Edmund Chada Baracat, Manoel Girão

\section{INTRODUCTION}

The literature is limited regarding reports of S. mansoni infection in the female genital tract. We report a case of a 44-years-old woman who underwent a laparoscopic momentum due to persistent pelvic pain. Histological examination of the specimen revealed an intramural leiomyoma with areas of hyaline and cystic degeneration. The leiomyoma contained many schistosoma eggs, consistent with S. mansoni, along with inflammation.

\section{CASE REPORT}

A 44-year-old woman from Lafaiete Coutinho (Bahia - Brazil), pregnant 2, para 1, arrived at our department with a 6-month history of pelvic pain and mild dysmenorrhea. The pelvic pain was moderate, continuous and progressive, and nonsteroidal anti-inflammatory agents did not relieve the associated dysmenorrhea. She reported no menstrual irregularity.

Her past medical history included asymptomatic uterine fibroids detected 10 years before that had not been considered as requiring treatment. Vulvar and vaginal inspection revealed no abnormalities. Bimanual examination of the pelvis showed an enlarged, irregular and tender uterus. Ultrasonographic examination was suggestive of uterine leiomyoma. Cervical smear and mammography were both within normal limits.

Due to a six-month persistent, low unremitting pelvic pain, the patient underwent laparoscopic myomectomy, which revealed an intramural fibroid, $10 \mathrm{~cm}$ in diameter. Its consistency was very soft and the cut surface had a yellowish, fading color, which seemed to be true fatty degeneration. No other intraoperative abnormalities were found.

Histological examination of the specimen revealed an

Department of Gynecology, Federal University of São Paulo-UNIFESP São Paulo/SP, Brazil

Email: analucia.arruda@terra.com.br intramural leiomyoma with areas of hyaline and cystic degeneration. The leiomyoma contained many schistosoma eggs, consistent with $S$. mansoni, along with inflammation (figure 1).

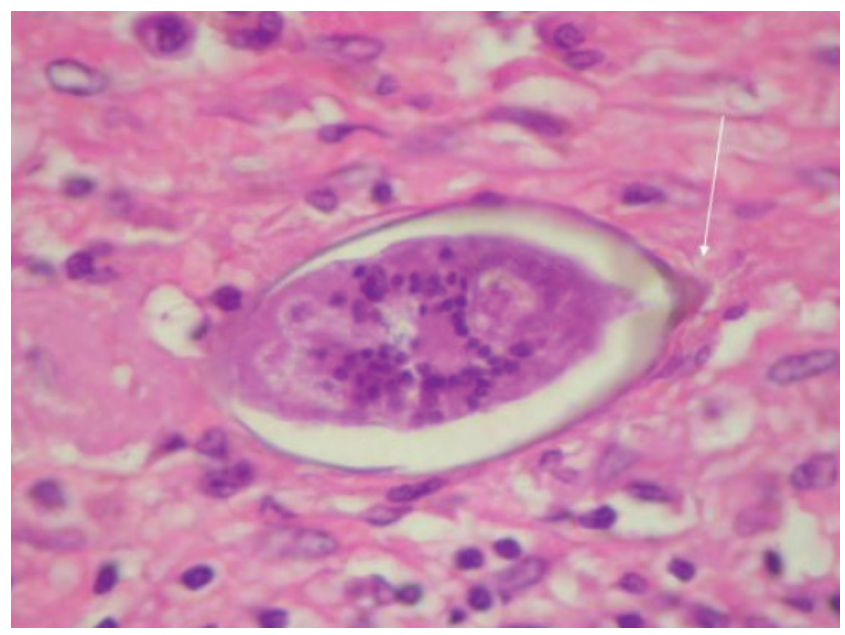

Figure 1 - (Hematoxylin and Eosin; 400X) S. Mansoni egg, with characteristic lateral spicule (arrow).

One week after laparoscopy the patient returned to our department and reported complete relief from the pelvic pain. The patient was treated with oral praziquantel after the result of the histological examination (40 days after laparoscopy). There were no residual symptoms and currently she remains free of symptoms.

\section{DISCUSSION}

The World Health Organization (WHO) estimates that $500-600$ million people in tropical and subtropical countries are at risk for schistosomiasis and over 200 million are infected. ${ }^{1}$ The species of major global importance are S. haematobium, S. mansoni and S. japonicum. ${ }^{2}$ S. mansoni is endemic to Brazil; the patient described in this report resides in Bahia, a state where S. mansoni infection is common. S. haematobium and S. japonicum have not been re- 
ported in Brazil. ${ }^{3}$ The most common site of $S$. mansoni infection is the intestinal tract. Prior cases of $S$. mansoni infection of the female genital tract have been described..$^{4-5}$ Histopathological studies have reported that $74 \%$ of female genital schistosomiasis infections were due to $S$. haematobium and $26 \%$ to $S$. mansoni ${ }^{4}$. In a Tanzanian village the prevalence of $S$. mansoni- related female genital schistosomiasis was $4 \%$ in a population where the prevalence of intestinal schistosomiasis was $14 \% .^{2-3}$

In the human body, the most common sites of $S$. mansoni infection are bowel venules. However, case reports of $S$. mansoni infection in the female genital tract have been described $^{4-5}$.In female genital schistosomiasis, eggs are most often found in the cervix and vaginal walls; less often eggs are seen in the ovaries and Fallopian tubes, and occasionally elsewhere in the genital tract. ${ }^{5}$

A possible factor that might contribute to this localization is the highly vascular nature of leiomyomas, suggesting embolization of schistosoma egg. Such embolization might have caused an inflammatory reaction with infarction, necrosis and pain. The inflammatory reaction led to degeneration of the myoma, thus justifying all the symptoms.

In schistosomiasis, most disease manifestations are due to eggs retained in host tissues. The granulomatous reaction around these eggs is cell-mediated and regulated by cytokine, cellular and humoral responses. Granuloma formation starts with recruitment of host inflammatory cells in response to antigens of the living organism within the eggs. Symptoms of acute schistosomiasis include fever, chills, headache, neck pain, diarrhea and arthromyalgia, but this inicial infection can also be asymptomatic.
Another mechanism that may contribute to the persistent pelvic pain is the presence of $S$. mansoni in venules of the intestinal or urinary tract, resulting in inflammatory reactions. We first considered the performance of serological tests, microscopic stool tests and collection of 24-h urine specimens; however, because the patient exhibited important relief from the symptom after surgery, we did not ask for these tests. However, we decided to treat her with oral praziquantel.

Schistosomiasis of the upper genital tract is usually an incidental finding. Ultrasound is inappropriate to determine the prevalence of lesions in the upper genital tract and the gold standard for such diagnosis is the demonstration of Schistosoma eggs through microscopical examination of body fluids or tissues. In this case, the diagnosis of schistosomiasis was not considered until histopathological tests were completed, and the entire pathological condition was originally attributed exclusively to the degeneration of the leiomyoma.

Uterine schistosomiasis can be associated with dysmenorrhea, chronic pelvic pain or masses, menstrual disorders, dyspareunia, infertility and pregnancy, as well as premature onset of labor and low birth weight. In the cervix, the symptoms or physical signs include post-coital bleeding, nodules, swelling, erosions, ulcers or tumors. ${ }^{5}$ It may also be an important risk factor for the spread of human immunodeficiency virus and human papilloma virus ${ }^{35}$. Though unusual, differential diagnosis of persistent pelvic pain should include Schistosoma infection in women who reside in or travel to tropical and subtropical countries.

\section{REFERENCES}

1. World Health Organization: Schistosomiasis Control. Division of Control of Tropical Diseases, 1998.

2. Leutscher P, Ravaoalimalala VE, Raharisolo C, Ramarokoto CE, Rasendramino M, Raobelison A. Clinical findings in female genital schistosomiasis in Madagascar. Trop. Med. Int. Health, 1998; 3 (4): $327-332$

3. Poggensee G, Kiwelu I, Weger V, Goppner D, Diedrich T, Krantz I, et al.Female genital schistosomiasis of the lower genital tract: prevalence and disease-associated morbidity in northern Tanzania. Journal of Infectious Diseases, 2000; 181 (3): 1210 - 1213.
4. Feldmeier H, Krantz I, Poggensee G. Female genital schistosomiasis as a risk- factor for the transmission of HIV. Int. J. STD $\mu$ AIDS, 1994; 5 (5): $368-372$.

5. Poggensee G, Krantz I, Feldmeier H. Female genital schistosomiasis: public health impact. Parasitol. Today, 1999; 15 (9): 378 - 381. 